\title{
EARLY CLASSIFICATION OF BRAIN TUMOR BASED ON IMAGE HISTOGRAM USING FUZZY-GENETIC ALGORITHM
}

\author{
Tamer Barakat, Mai Elbadwey, and Khaled H . Ibrahim \\ Electrical Engineering Department, Faculty of Engineering, Fayoum University \\ Fayoum - Egypt. \\ tmb00@fayoum.edu.eg \\ me1523@fayoum.edu.eg \\ Khi00@fayoum.edu.eg
}

\begin{abstract}
A Brain Tumor Classification Framework has been outlined and created. The framework uses computer based strategies to identify tumor blocks or lesions and classify the sort of tumor utilizing matching histogram in MRI images of different patients with brain tumors. The picture processing methods such as picture segmentation, picture enhancement. Several techniques can classify the tumor such as Support vector machine (SVM), artificial neural networks (ANN), and Naive Bayes, but they did not accomplish the required accuracy. The automatic classification of tumors requires high precision since the non-accurate conclusion would cause a rise within the predominance of more serious diseases. In this paper, the proposed method using fuzzy logic and genetic algorithm based on image histogram to enhance the brain tumor classification. The experimental result showed that our technique is more effective than the previous techniques, as well as the classification accuracy efficiency is $99.9 \%$.
\end{abstract}

Keywords: Image Segmentation, Histogram, MRI, Fuzzy, membership and weight.

\section{Introduction}

Automated classification and discovery of tumors in several medical pictures requests high precision since it deals with human life. Too, computer help is highly looked for in medical institutions due to the reality that it seem improve the results. It has been proven that double reading of medical pictures may lead to way better tumor discovery. But the cost caused in reading is very high; hence great computer program to help people in medical institutions is of great interest these days.

Magnetic resonance images (MRI) combine a common advantage with CT - high spatial resolution pictures - but with no ionizing radiation presentation, which makes it a more secure method. MRI holds a really particular place among imaging modalities, since it is highly flexible from particular centres of see ,and it offers flexibility of slice positioning at any point inside the three dimensional space, included to an really great soft tissue differentiate.
Classification of brain tumors is a more difficult task due to frequent change of behavior for tumor region's texture, form, discontinuity, irregularity and uncertain boarder of gliomas. Analyzing the medical images manually gives more mistakes, as well as takes a long time. This problem significantly degrades the accuracy of the classifier and has a negative effect on computational time. The hybrid classification technique to enhance the accuracy problem. The proposed methode consists of sub steps: FCM segmentation based on otsu's thresholding, the image histogram and fuzzy-genetic classifier. FCM segmentation is an unsupervised classification algorithm [14]. Otsu's Thresholding is used for removing the noise of the picture to get a high-quality image without losing any important information. It is a critical technique in medical picture preparing. The basic idea is to calculate the optimal threshold gray level value required to isolate the object region from the overall 
image. This algorithm greatly improves the speed of thresholding and has superior resistance to salt and pepper noise [13].

In the area of medical diagnosis, some Artificial Intelligence (AI) methods including neural networks and fuzzy logic are successfully applied to a wide variety of decision-making problems. In the proposed method, the data set BRATS 2013 which is online available is used [8]. It comprises of low-grade gliomas (LGG) and high-grade gliomas (HGG).

MRI sequences are T1-weighted, T2weighted, and Fluid Attenuated Inversion Recovery (Flair) scans. Flair has a better resolution since it involves 1019 images for HGG type and 206 images for LGG type.

MRI images are converted into grayscale images, and then they are segmented using the FCM method biased on Otsu's thresholding. The proposed classification method generates fuzzy if-then rules based on image histogram, and then optimizes the matching accuracy using genetic algorithm.

The rest of this paper is organized as follows. Section 2 describes the related work for brain tumor classification. Section 3 presents the proposed model. The experimented results obtained by the proposed method and discussions are given in section 4. Finally, the conclusion.

\section{Related work}

Saumya Chauhan et al. [1] detect the tumor from MRI pictures utilizing Instance-based K-Nearest utilizing Log and Gaussian Weight Kernels (IBKLG).Unimportant information from the picture is evacuated utilizing median filtering. The input MRI pictures are Grayscale. HSV colour-map is utilized to change over the picture into an RGB picture, which makes a difference to imagine the colour contrasts. K-means clustering calculation is connected to make sections of the picture. Texture Features are extricated. This method achieves a better accuracy with $86.6 \%$ compared to Naïve Bays.
Garima Singh et al. [2] detect the brain tumors using K-Means Segmentation and Normalized Histogram. They classify using the Naïve Bayes method and SVM into normal and abnormal.

The results were not satisfactory and the classification of brain tumors was not accurate. However, G Rajesh Chandra et al. [3] make a segmentation method using soft thresholding Discrete Wavelet Transform (DWT) and genetic algorithm for enhancement of the image. This method achieved SNR (signal to noise ratio) value from 20 to 44 and segmentation accuracy from $82 \%$ to $97 \%$ based on ground truth MRI images.

Anitta Antony et al. [4] classify the tumor as meningioma or glioma. They segment the image into three clusters $\mathrm{K}=3$ and extract 1024 features using weighted Neighbor Distance using Compound Hierarchy of Algorithms Representing Morphology (Wndchrm tool)with SVM classification. An $80 \%$ accuracy is achieved, however for 154 features with SVM classification, 93.33\% accuracy is achieved.

Saddam $\mathrm{H}$ et al. [5] utilize cascade deep convolutional neural networks (CNN) design for tumor segmentation. This strategy joined both local and global features since is important for tumor segmentation. The utilize of max-pooling, max-out and drop-out complement the learning handle, progressing training and testing speed by decreasing highlights in a completely associated layer as well as decreasing the number of parameters, which decrease the chances of over-fitting.

Reema Mathew et al. [6] design a K-mean clustering algorithm for image segmentation and extracted from it GLCM, DWT and Gabor wavelet features. Since the vector gotten could be huge, it is decreased with the assistance of Principal component analysis (PCA). PCA output is the input for the SVM classifier. Their results appeared that the SVM method with a linear kernel achieves more precision for a large number of the data. Sudipta Roy et al, [7] develop a classifier system using ANFIS for brain tumor tissue. 
It got $98.25 \%$ precision on the Harvard benchmark dataset. Examination with a huge number of variables requires a huge amount of memory and computation control. Feature extraction may be a term for strategies of building combinations of the factors to get around these problems while portraying the information with adequate exactness.

\section{Proposed Method}

In this study the design of the proposed system provides the MRIs with accurate detection and classification into a benign and malignant .The proposed classification method consists of sub steps as shown in fig.1.

The image pre-processing has the objective of removing the noise and keeping the information for the tumor by thresholding. In the FCM segmentation, the tumor is extracted from the image with high accuracy. In the next stage, the histogram from the segmented image. The final stage is the classification based on the image histogram using fuzzy system. The matching accuracy is optimized using genetic algorithm.

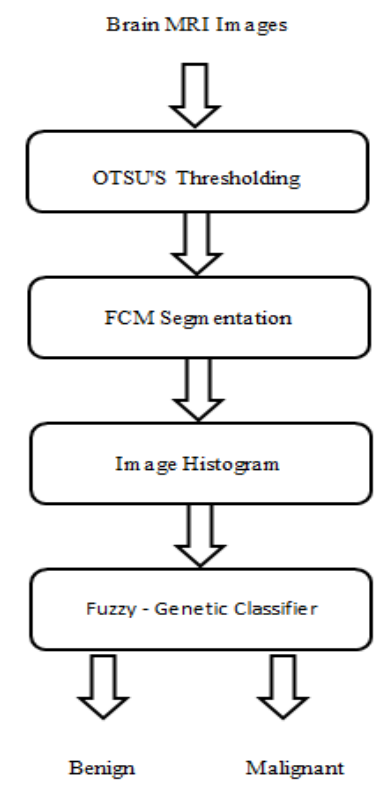

Fig. 1 Flowchart of the proposed method for Brain Tumor Classification

\subsection{Proposed Image Pre-processing}

The goal of pre-processing is striding the image data that smother undesired distortions and improves image features that are pertinent for processing $[9,10]$.

It includes sub steps such as thresholding and FCM segmentation $[11,12]$.

The flow chart of the proposed method is shown in fig.2. It has higher accuracy than other methods such as neural network or Kmean method. FCM clustering for brain tumor gives three clusters; CSF, WM, and GM (brain tissues) $[15,16]$.

The fuzzy partition should have two properties; homogeneity within the clusters and heterogeneity between clusters $[17,18]$. Utilizing an averaging (Gaussian) filter and a median filter to remove salt and pepper noise from an image.

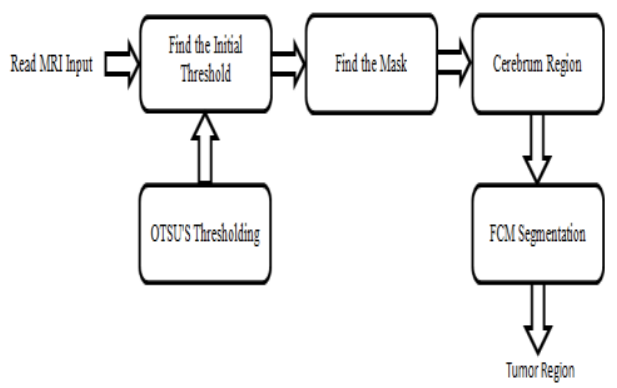

Fig. 2 Flowchart of the proposed method for image segmentation

\subsection{Image Histogram}

Histograms are an effective strategy to depict distributions of continuous factors. Histograms plot the frequency of event of an observation within the intervals. It can be respected as a sort of classification of information. Each sample is sorted into one of several "bins" agreeing to some property.

Firstly, we assess the number of bins 'S', used to build MRI image histograms, on the classification results. The main aim from our proposed system is to obtain a feature space, 
which may be utilized to discriminate between benign and malignant tumor.

\subsection{Fuzzy classifier}

In later decades utilizing fuzzy theory in administration and building has increased significantly. Fuzzy science is able to develop models which can handle qualitative data intelligently nearly like a human. Data input for the fuzzy system is the histogram from the segmented image. Fuzzy systems use the linguistic variables to create choices based on fuzzy rules and this is the reason why these systems get better results compared to those use crisp values [19-21]. Thus, fuzzy logic provides simple modeling, fast convergence, and noise reduction. The proposed fuzzy system could be illustrated through the following items: linguistic variables, fuzzification, knowledgebase, fuzzy logic operations, and defuzzification.

\subsubsection{Fuzzification}

In this phase, the input is the histogram from the segmented image and converts this crisp value to a fuzzy weight depending on the fuzzy membership. The membership functions have multiple types such as triangular, Gaussian, trapezoidal, piecewise linear and singleton. In the proposed fuzzy system, two output fuzzy sets are labeled "low" and "High".

The MF is the triangular membership function. A triangular MF is indicated by three parameters $\{a, b, c\}$ as takes after:

The parameters $\{a, b, c\}$ (with $a<b<c)$ specify the $\mathrm{x}$ coordinates of the three corners of the TMF. Triangular memberships function $(a, b, c)$ by calculation in equation (1).

$$
\mu_{A}(x)\left\{\begin{array}{lr}
0 & x \leq a \\
\frac{x-a}{b-a} & a<x \leq b \\
\frac{c-x}{c-b} & b<x<c \\
0 & x \geq c
\end{array}\right.
$$

\subsubsection{Histogram Matching}

Histogram-based picture coordinatin calculations attempt to measure the similarity in contents through their histograms between a model picture and any pictures in database, i.e., target images, in arrange to properly classify or recover images. Histogram intersection (HI) is calculated by equation (2).

Where,

$$
\text { (2) } \quad H I=\sum_{i=1}^{n} \min \left(h_{M}(i), h_{T}(i)\right)
$$

$\mathbf{h}_{\mathbf{M}}$ The histogram of a model image.

$\mathbf{h}_{\mathbf{T}}$ The histogram of a target image.

n Number of bins

The intersection of two fuzzy sets High and Low has the triangular membership function. Applying a binary operation on the unit interval function $[0,1]$.

The shaded area from the intersection between the test histogram and the triangular membership

function is the $\mu_{\mathbf{h}}$ or $\mu_{\mathbf{I}}$ show in fig.3, and then apply the fuzzy rule base for $\mu_{\mathbf{h}}$ and $\mu_{\mathbf{I}}$ values. It is cleared from fig.3, that $\mu_{\mathbf{h}}>\mu_{\mathbf{l}}$. So that the image histogram belong to high and the tumor is malignant.

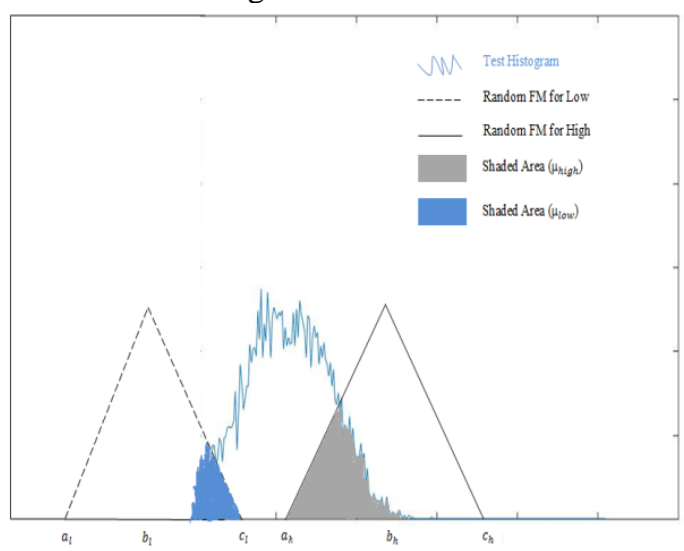

Fig. 3 the histogram matching between the test histogram and fuzzy membership

\subsubsection{Fuzzy Rule base}

The proposed rule base for fuzzy system is shown in fig.4. 


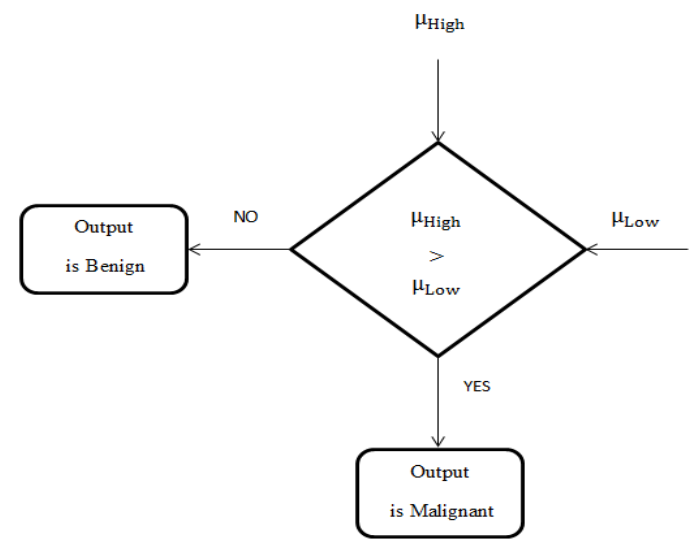

Fig.4 Flow chart of the proposed fuzzy rule

\subsection{Genetic Algorithm}

The proposed GFS (Genetic Fuzzy System) is a fuzzy system classifier improved by a genetic algorithm. In the proposed genetic algorithm, the parameters $(a, b, c)$ for each fuzzy membership are optimized such that the classification accuracy is as maximum as possible [22]. The fitness function of the proposed Genetic algorithm is the classification accuracy that could be calculated by equation (3).

$$
A C=\frac{T_{B}+T_{M}}{T_{B}+T_{M}+F_{B}+F_{M}} * 100
$$

Where,

$\mathbf{T}_{\mathbf{B}}$ Number of true benign.

$\mathbf{T}_{\mathbf{M}}$ Number of true malignant.

$\mathbf{F}_{B}$ Number of false benign.

$\mathbf{F}_{\mathbf{M}}$ Number of false malignant.

The parameters $(a, b, c)$ defining the fuzzy membership functions are defined as a chromosome in the optimisation process as shown in fig.5. A group of chromosomes (a population) is created at random. A simulation evaluates the fitness of each chromosome. Then, based on the results of the simulation, the population evolves through three genetic operations: selection, crossover and mutation. The phase of assessment-evolution is repeated until a condition is reached. The false positive error rates and false negative error rates are used to determine the fitness of a chromosome when improving the fuzzy system used for classification.

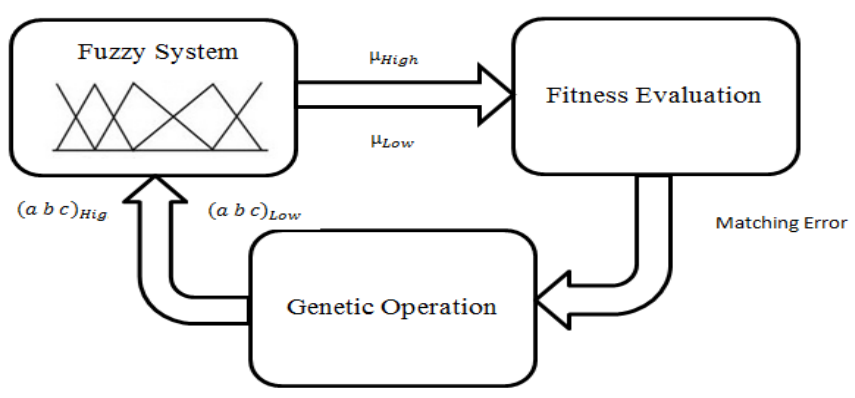

Fig.5 Fuzzy system optimization by combining a genetic algorithm

\section{RESULTS}

This section presents in details the simulation of the proposed methods that carried out on the BRATS2013 dataset. The proposed method is implemented in MATLAB R2016a and executed in a PC with AMB A8 -5545M APU processor with $1.70 \mathrm{GHz}$ speed and 8 GB of RAM.

\subsection{Results of proposed image processing}

\subsubsection{FCM segmentation based on}

\section{OTSU's thresholding}

In this stage, there are two sub-steps are Otsu's

thresholding method and FCM segmentation. The obtained FCM parameters are shown in Table 1.

\section{Table 1 FCM system parameters}

\begin{tabular}{|c|c|}
\hline Max. No. of iteration & limited to100 \\
\hline Min. improvement & 1e-5 is an optional limit. \\
\hline No. of clusters & 3 \\
\hline
\end{tabular}

\subsubsection{Otsu's thresholding}

Firstly, Original image is converted into gray scale image. Fig. 6 shows both of the original images and enhanced one.

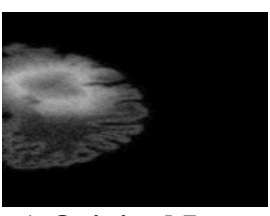

a) Original Image

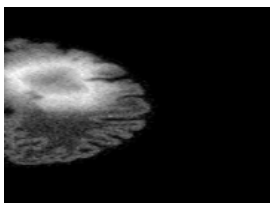

b) Enhanced Image

Fig.6 a. Original, b. Enhanced Image 
4.2 Image Histogram for the segmented image
Table 2 shows the histogram for each MRI image and its type.

Table 2 the segmented images and image histogram.

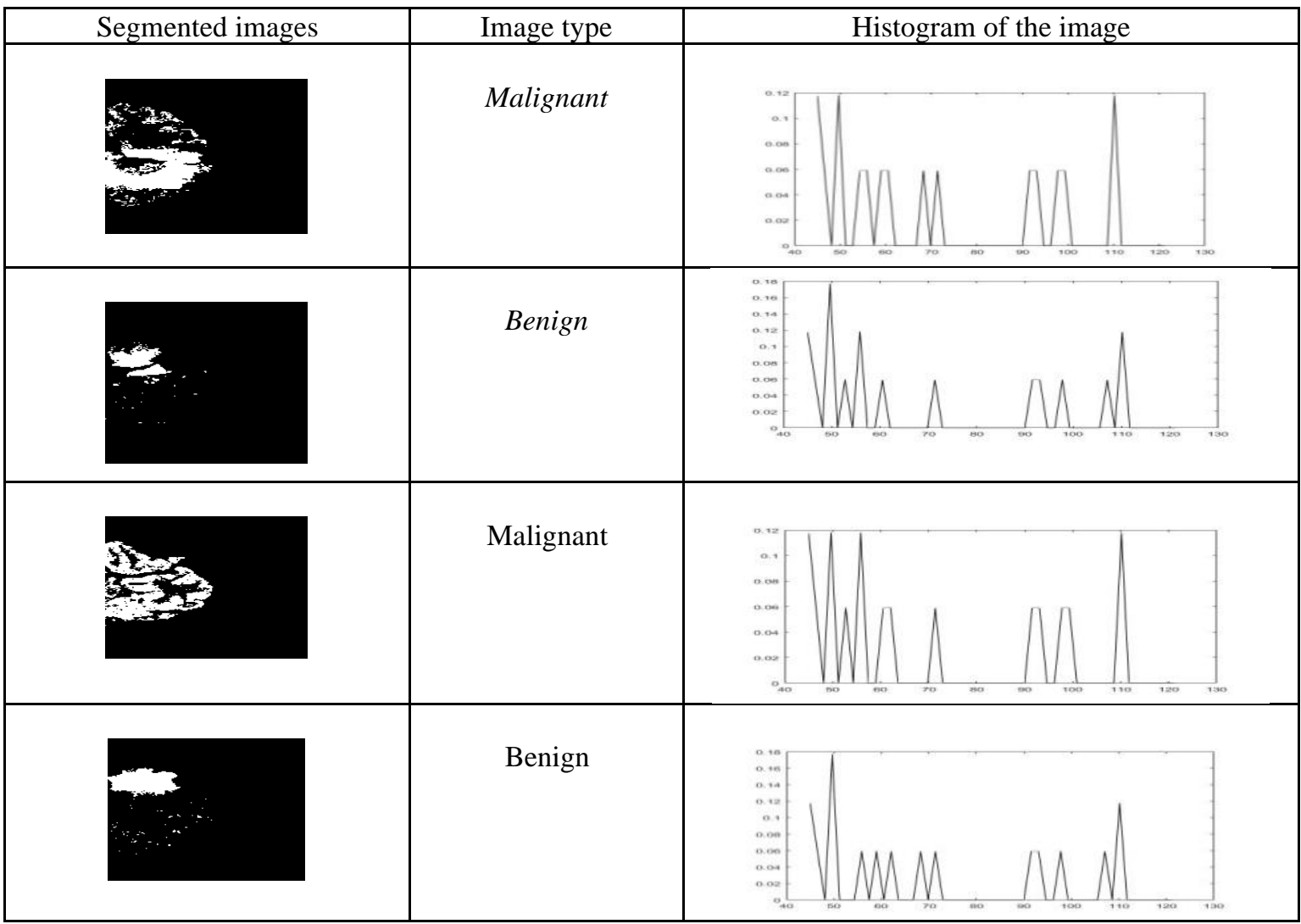

\subsubsection{Fuzzy c-means clustering}

The FCM algorithm gives the best results.It divides the input image into 3 clusters and calculates the center for each cluster 3 Levels are: $\{0.2843,0.1605,0.5237\}$. Fig.7 shows the result from FCM segmentation and the tumor region.

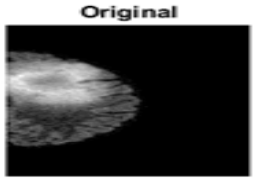

FCMO,level=0.152500

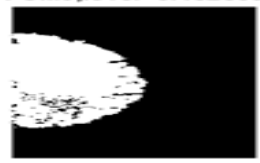

Fig.7 the result of the proposed 3 class thresholding FCM Segmentation. 
In this study, the counts and pixels value (ranges) for each histogram are the input for the classification stage.

\subsection{Genetic Algorithm}

GA is used to get the optimal fitness value and get higher accuracy value. The parameters of the proposed Genetic fuzzy system are shown in Table3.

Table 3.The proposed genetic Parameters

\begin{tabular}{|r|c|}
\hline No. of genes & 6 \\
\hline No. of Chromosomes & 100 \\
\hline Crossover probability & 0.6 \\
\hline mutation Probability & 0.01 \\
\hline stopping crite & 30 \\
\hline
\end{tabular}

Table 4 shows the best parameters for TFN (triangular fuzzy numbers).

The proposed classification method using fuzzy logic and genetic algorithm is achieved accuracy $99.9 \%$.

Table4. The results from Genetic Fuzzy system

\begin{tabular}{|c|c|c|c|}
\hline Parameters & A & B & c \\
\hline High & 70 & 75 & 80 \\
\hline Low & 88 & 93 & 98 \\
\hline
\end{tabular}

\section{Conclusion}

The proposed system is performed on different MRI images without manual intervention. It achieves better results compared to other methods. The pre-processing and FCM segmentation are used to enhance the image and to separate the interested region ( Tumor region) from the normal tissues GM, WM and CSF. Then calculates the histogram for each segmented image. Finally, the classification stage is implemented with fuzzy-genetic system. The proposed hybrid method achieved higher accuracy than the other methods such as: Support vector machine (SVM), artificial neural networks (ANN), and Naive Bayes. The classification accuracy obtained for this system is $99.9 \%$.

\section{References}

[1] Saumya Chauhan, Aayushi More, Ritumbhra Uikey, Pooja Malviya, Asmita Moghe"Brain Tumor Detection and Classification in MRI Images using Image and Data Mining" ISBN 978-15090-4760-4/17/\$31.00@2017 IEEE

[2] Garima Singh, Dr. M.A. Ansari"Efficient Detection of Brain Tumor from MRIs Using K-Means Segmentation and Normalized Histogram"978-1-4673-69848/16/\$31.00 @ 2016 IEEE

[3] G Rajesh Chandra, Dr.Kolasani Ramchand H Rao" TUMOR DETECTION IN BRAIN USING GENETIC ALGORITHM" ELSEVIER2016

[4] Anitta Antony, Ahammed Muneer K V"Feature Optimization and Classification of Tumor Grades using MR Images"978-1-5386-49664/18/\$31.00 @2018 IEEE

[5] Saddam Hussain, Syed Muhammad Anwar, Muhammad Majid"Brain TumorSegmentationusingCascadedDe epConvolutionalNeuralNetwork"9781-5090-2809-2/17/\$31.00 @2017 IEEE

[6] Reema Mathew A, Dr. Babu Anto P, Thara N.K"Brain Tumor Segmentation And Classification using DWT, Gabour Wavelet And GLCM"978-1-5090-6106-8/17/\$31.00 C)2017 IEEE 2017

[7] SudiptaRoy, Shayak Sadhu1, Samir Kumar Bandyopadhyay,Debnath Bhattacharyya and Tai-Hoon Kim" Brain Tumor Classification using Adaptive Neuro-Fuzzy Inference System from MRI" 2016 SERSC.

[8] International MICCAI BraTS Challenge. Proceedings of the 6th MICCAI BraTS Challenge (2017)

[9] Media Hadi Avizenna, Indah Soesanti, Igi Ardiyanto" Classification of Brain Magnetic Resonance Images Based on Statistical Texture"978-15386-7599-1/18/\$31.00 @2018 IEEE 
[10] Nidhi Gupta, Pushpraj Bhatele, Pritee Khanna " Glioma detection on brain MRIs using texture and morphological features with ensemble learning " ELSEVIER2019

[11] Suraj S. Gawande, Ms. Vrushali Mendre" BRAIN TUMOR DIAGNOSISUSING IMAGE PROCESSING: A SURVEY"978-15090-3704-9/17/\$31.00 @ 2017 IEEE

[12] Swapnil R. Telrandhe, Amit Pimpalkar, Ankita Kendra" Detection of Brain Tumor from MRI images by using Segmentation \&SVM"978-14673-9214-3/16/\$31.00 @ 2016 IEEE.

[13] Sen Qian, Guirong Weng"Medical Image Segmentation Based onFCMAndLevelSetAlgorithm"978-14673-9904-3/16/\$31.00 @2016 IEEE

[14] Nidhi Gupta, Pushpraj Bhatele, Pritee Khanna "Identification of Gliomas from brain MRI through adaptive segmentation and run length of centralized patterns" ELSEVIER2017

[15] M.G.Sumithra, B.Deepa" Performance Analysis of Various Segmentation Techniques for Detection of Brain Abnormality"978-1-50902597-8/16/\$31.00c 2016 IEEE

[16] Mohit Khandelwal, Shraddha Shirsagar, Paresh Rawat"MRI Image Segmentation using Thresholding with 3-class C-means Clustering"978-15386-0807-4/18/\$31.00 @2018 IEEE
[17] Hong Huang, Fanzhi Meng, Shaohua Zhou1, Feng Jiang, Gunasekaran Manogaran" Brain image segmentation based on FCM clustering algorithm and rough set"2169-3536 (c) 2018 IEEE.

[18] Min Li1, Limei Zhang, Zhikang Xiang, Edward Castillo, Thomas Guerrero"An Improved Fuzzy CMeans Algorithm for Brain MRI Image Segmentation"978-1-5090-34840/16/\$31.00 @2016 IEEE

[19] Mrs. Meenakshi M. Pawar, Dr. Sanjay N. Talbar "Genetic Fuzzy System (GFS) based Wavelet Cooccurrence Feature selection in Mammogram Classification for Breast Cancer Diagnosis"2016

[20] Salma Elhag, Alberto Fernández, Abdullah Bawakid, Saleh Alshomrani, Francisco Herrera" On the combination of genetic fuzzy systems and pairwise learning for improving detection rates on Intrusion Detection Systems" ELSEVIER2015

[21] Adriano S. Koshiyama, Marley M.B.R. Velasco, Ricardo Tanscheit" GPFIS-CLASS: A Genetic Fuzzy System Based on Genetic Programming for classification" ELSEIVER2015

[22] M.E. Cintra a, H.A. Camargo, M.C. Monard "Genetic generation of fuzzy systems with rule extraction using formal concept analysis" ELSEVIER2016 


\section{التصنيف المبكر لورم الدماغ بناء على الرسم البيانى للصورة بأستخدام خوارزمة ضبابية وراثية}

تم أنثاء نظام عمل لتصنيف ورم الدماغ و يستخدم هذا النظام أستراتيجات تعتمد على الكمبيوتر لتحديد كتلة الورم وتصنيف نوع التواء

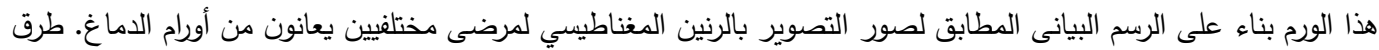

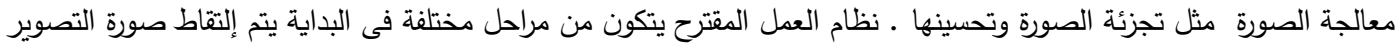

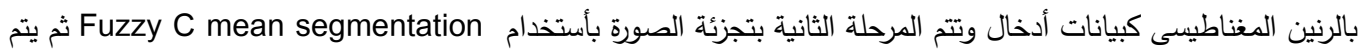
استخراج الورم من الصورة ـ أخيرًا يُصنف الورم على أنه حميد أو خبيث باستخدام نظام ضبابي وراثي يعتمد على الرسم البياني.

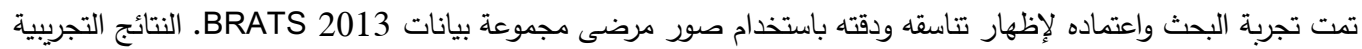
حقت دقة تصنيف 100٪ 